\title{
Minimum Copper Loss Flux-Weakening Control of Surface Mounted Permanent Magnet Synchronous Motors
}

\author{
Jiunn-Jiang Chen and Kan-Ping Chin, Member, IEEE
}

\begin{abstract}
This study presents a novel current loop design method capable of automatic flux-weakening control with minimum copper loss for surface mounted permanent magnet synchronous motors (SPMSM). The proposed current controller can automatically re-compute the d-axis current command to defer output voltage saturation. Consequently, the motor operations in the flux-weakening region are also contained in the stable operating region. Analysis results indicate that since the output voltage vector in the flux-weakening region produced by this controller is consistently on the boundary of the maximum output voltage vector allowed by the inverter, the corresponding flux-weakening current is the optimal value in the sense of minimum copper loss. This minimum copper loss design differs from the maximum output torque design and the constant power design of the flux-weakening control methods found in the literature. Experimental results further demonstrate the feasibility of the proposed current controller and its ability to maximize the speed range of the motor drive for a given inverter capacity.
\end{abstract}

Index Terms-Current control, flux-weakening control, PMSM, torque.

\section{INTRODUCTION}

$\mathbf{P}$ ERMANENT magnet synchronous motors (PMSM) have recently been widely adopted for servo drives in industrial applications, because of their numerous attractive features such as high efficiency, superior power density, and high torque to inertia ratio. However, the servo controlled PMSMs have a limited operating range, owing to the control strategies applied and the inverter capacities installed. An inverter's capacity is fixed during motor operations. As a result, how to apply control strategies to effectively expand the operating range under a fixed inverter capacity is an interesting topic in the field of PMSM controls. The output voltage limit of the inverter is generally a major limitation to expanding the operating range of PMSM controls. As the back emf rises with an increasing speed, the back emf cancels a portion of the input voltage, which is bound by the inverter capacity, and limits the current injected to the motor, thus limiting the maximum speed of the PMSM. To extend the operating speed range of PMSMs, numerous algorithms were proposed to accomplish flux-weakening control [1]-[7]. Employing a negative d-axis current to

Manuscript received May 21, 2001; revised March 1, 2003. Recommended by Associate Editor P. C. Luk.

J.-J. Chen is with Aetas System Incorporated Company, Hsinchu, Taiwan, R.O.C. (e-mail: jjchen.me84g@nctu.edu.tw; justin.chen@aetas.com.tw).

K.-P. Chin, deceased, was with the Department of Mechanical Engineering, National Chiao-Tung University, Hsinchu 300, Taiwan, R.O.C.

Digital Object Identifier 10.1109/TPEL.2003.813751 suppress the rising back emf is a common characteristic of these flux-weakening control strategies for PMSMs. The negative $\mathrm{d}$-axis current is the so-called flux-weakening current, while the extended operating region achieved by flux-weakening control is the so-called flux-weakening region. References [1]-[4] proposed several flux-weakening controls of various PMSMs, including surface mounted permanent magnet synchronous motor (SPMSM) and interior permanent magnet synchronous motor (IPMSM). These methods design flux-weakening current command schemes from the perspective of maximum torque output or constant power output under steady-state analyzes. However, these methods are extremely sensitive to the motor parameters used to compute the flux-weakening current command and the switching velocity. Moreover, even if the flux-weakening current command computed from imprecise motor parameters can still perform flux-weakening control, the original intention is violated and the operating range is reduced. Existing investigations [5]-[7] have presented flux-weakening controls of PMSMs that do not require prior knowledge of motor parameters. One of the methods proposed that the flux-weakening current command should be proportional to the filtered q-axis current error [5]. This method is inherently instable, because the existence of the q-axis current error in the flux-weakening region is required to maintain the generation of the flux-weakening current command. Another method indicated that the flux-weakening current command be adjusted through a proportional-integral (P-I) computation of the voltage error between the maximum output voltage vector and the output voltage vector of the current controller [6], [7]. This method can theoretically achieve effective flux-wakening control because it attempts to stop voltage saturation and expand the operating range. However, the voltage commands produced by this method must pass through the saturated voltage region. Consequently, the original output commands produced by the current control loop must be replaced by commands modified by the overmodulation strategy. This result effectively changes the current controller to a new form with unknown stability and performance characteristics, and may not achieve the original design objectives. Therefore, PI gains must be selected appropriately to ensure stability in the voltage saturation region.

Flux-weakening control should aim primarily to defer voltage saturation and expand the operating range. This study proposes a novel flux-weakening control method that differs from existing flux-weakening control methods in that it is integrated into the current loop design. The proposed current controller can 
automatically re-compute the d-axis current command to defer output voltage saturation, so that the current control loops in the normal operation region $\left(i_{d}=0\right)$ and in the flux-weakening operation region $\left(i_{d}<0\right)$ are coherent. Accordingly, control stability and performance can be guaranteed even in the flux-weakening region. Furthermore, because the output voltage vector of the current controller is always on the boundary of the maximum output voltage vector during operations in the flux-weakening region, the corresponding flux-weakening current is insensitive to the motor parameters used in the controller and will be the optimal value for minimizing copper loss [8]. This design feature differs from the maximum output torque design and the constant power design [1]-[4].

The rest of this paper is organized as follows. Section II presents the mathematical model of a SPMSM. Section III then reviews and discusses why flux-weakening control is needed to extend the operating range of motor control under a fixed inverter capacity, and also discusses how flux-weakening control can be achieved while minimizing copper loss. Meanwhile, Section IV describes the proposed current controller and its implementation technique, and Section V summarizes the experimental results. Finally, conclusions are made in Section VI.

\section{MOTOR MODEL}

The mathematical model of the electrical subsystem of a SPMSM in a synchronous frame, or the so-called $d-q$ frame, can be described as

$$
\begin{aligned}
\frac{d}{d t}\left\{\begin{array}{l}
i_{d} \\
i_{q}
\end{array}\right\}= & {\left[\begin{array}{cc}
-\frac{R}{L} & N \omega \\
-N \omega & -\frac{R}{L}
\end{array}\right]\left\{\begin{array}{l}
i_{d} \\
i_{q}
\end{array}\right\} } \\
& -\frac{1}{L}\left\{\begin{array}{c}
0 \\
K N \omega
\end{array}\right\}+\frac{1}{L}\left\{\begin{array}{l}
v_{d} \\
v_{q}
\end{array}\right\} \\
T_{e}= & \frac{3}{2} K N i_{q}
\end{aligned}
$$

where $\left\{i_{d} i_{q}\right\}^{T}$ denotes the electrical state vector whose components are the stator currents in the $d-q$ frame; $\left\{v_{d} v_{q}\right\}^{T}$ denotes the input vector whose components are input voltages in the $d-q$ frame; $\omega$ is the rotor velocity; $L$ is the phase inductance; $R$ denotes the phase resistance; $N$ is the number of pole pairs; $K$ represents the torque (permanent magnet) constant; and $T_{e}$ denotes the electromagnetic output torque.

\section{REVIEW OF FLUX-WEAKENING CONTROL}

This section discusses the theoretical steady-state operable region of motor drives with a fixed inverter capacity and the essentials of flux-weakening controls. Moreover, the conditions required to accomplish flux-weakening control while minimizing copper loss are also revealed.

\section{A. Theoretical Steady-State Operable Region}

Inverter capacity is generally fixed during motor controls. Inverted capacity, including voltage and current constraints, can be expressed as

$$
\begin{aligned}
i_{d}^{2}+i_{q}^{2} & \leq I_{\max }^{2} \\
v_{d}^{2}+v_{q}^{2} & \leq V_{\max }^{2}
\end{aligned}
$$

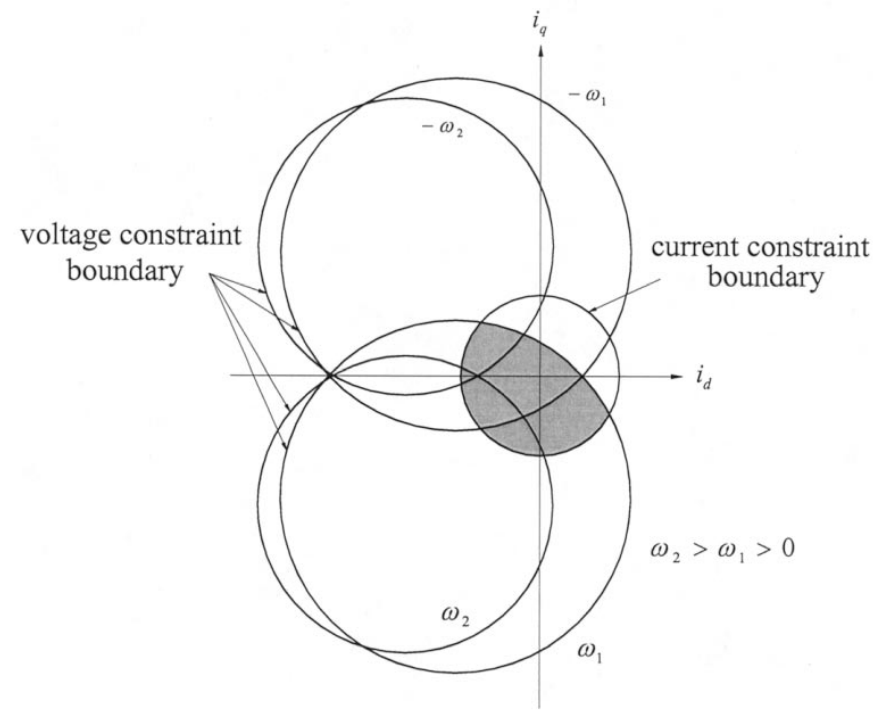

Fig. 1. Theoretical steady-state operable region expressed on the $i_{d}-i_{q}$ plane.

The current constraint (3) stipulates that the magnitude of the inverter output current vector can not exceed the maximum current, $I_{\max }$, while the voltage constraint (4) stipulates that the magnitude of the inverter output voltage vector can not exceed the maximum voltage, $V_{\max }$. Generally, $I_{\max }$ is determined by the inverter current rating and is constant. Meanwhile, $V_{\max }$ is determined by the dc link voltage, $V_{D C}$, and forms a hexagon in the voltage vector space if a space vector pulse width modulation (SVPWM) strategy is applied. For convenience, the inside tangent circle of the hexagon is commonly used to replace the hexagon as the voltage limit. Consequently, the maximum voltage can be expressed as $V_{\max }=V_{D C} / \sqrt{3}$.

The theoretical steady-state operable region of motor control under a fixed inverter capacity can be completely expressed by $i_{d}$ and $i_{q}$. Assuming that the motor has reached its steady-state, the voltage equations of the motor model can be expressed as

$$
\begin{aligned}
& v_{d}=R i_{d}-L N \omega i_{q} \\
& v_{q}=L N \omega i_{d}+R i_{q}+K N \omega .
\end{aligned}
$$

By substituting (5) and (6) into (4), the voltage constraint can be re-expressed as

$$
\begin{gathered}
\left(i_{d}+\frac{K L N^{2} \omega^{2}}{R^{2}+L^{2} N^{2} \omega^{2}}\right)^{2}+\left(i_{q}+\frac{K N \omega R}{R^{2}+L^{2} N^{2} \omega^{2}}\right)^{2} \\
\leq \frac{V_{\max }^{2}}{R^{2}+L^{2} N^{2} \omega^{2}}
\end{gathered}
$$

Given a designated operating speed, $\omega$, and a fixed inverter capacity, $I_{\max }$ and $V_{\max }$, the two constraints (3) and (7) can be plotted together on an $i_{d}-i_{q}$ plane, as displayed in Fig. 1 . Because (7) contains the variable $\omega$, the voltage constraint area varies with operating speed. Actually, as illustrated in Fig. 1, the voltage constraint circle shrinks and moves to the left as the designated operating speed increases. On the other hand, the current constraint is not dependent on operating speed and the current constraint circle remains unchanged in the $i_{d}-i_{q}$ plane. Notably, unlike the figures of general flux weakening analyses presented in [1]-[3], Fig. 1 does not ignore the effect of stator 


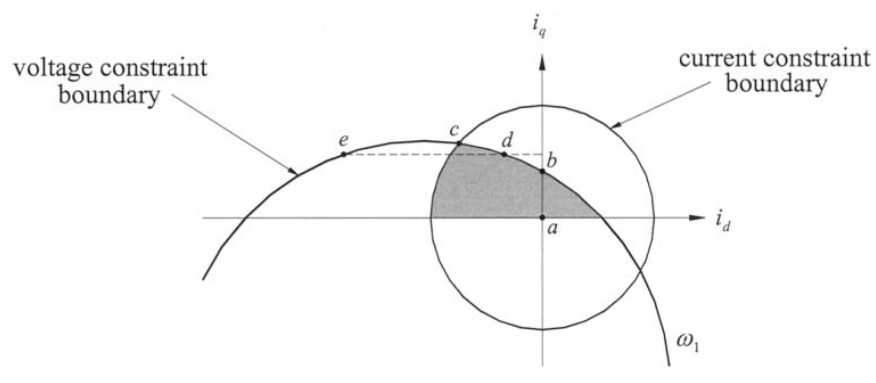

Fig. 2. Minimum copper loss design.

resistance. The area within the intersection of the current and voltage circles corresponds to the solutions that satisfy both inequalities (3) and (7). This area is the theoretical steady-state operable region expressed on the $i_{d}-i_{q}$ plane for the designated operating speed and the inverter capacity. For example, the shadowed area in Fig. 1 denotes the theoretical steady-state operable region for the operating speed, $\omega_{1}$.

In addition to clearly indicating the operable region, Fig. 1 also shows the necessity of performing flux-weakening control to extend the operating range of the motor control. For SPMSMs, because the d-axis current does not generate an electromagnetic torque, as expressed in (2), it is usually controlled to zero under normal operations to reduce copper loss from winding resistance. However, according to Fig. 1, the regions where $i_{d}=0$ are not always within the operable region, especially when the motor is operating with a high load torque, large $i_{q}$, or/and high speed. Under these circumstances, the d-axis current, $i_{d}$, must be maintained at a negative value to shift the operating point laterally into the operable region (shadowed area in Fig. 1). The negative d-axis current is the so-called flux-weakening current, and the flux-weakening control is responsible for driving the flux-weakening current such that the motor always operates inside the operable region even when operating conditions vary.

\section{B. Minimum Copper Loss}

To simplify the following analyzes, Fig. 1 is re-plotted to Fig. 2, which only contains the current constraint circle and voltage constraint circle for a single speed $\omega_{1}$. Furthermore, the shadowed area of the operable region in Fig. 2 represents motor operation when $\omega i_{q} \geq 0$. In Fig. 2, line segment $a b$ and arc $b c$ denote the minimum copper loss regions of the operable range, because operating points in these regions have the smallest magnitude of $i_{d}$ for a given $i_{q}$. Line segment $a b$ belongs to the normal operating region $\left(i_{d}=0\right)$, while arc $b c$, which is on the voltage constraint boundary, belongs to the flux-weakening operating region $\left(i_{d}<0\right)$. Consequently, a necessary condition to minimizing copper loss in a flux-weakening operation is to keep the output voltage vector of the current controller on the voltage constraint boundary in the flux-weakening region. However, if voltage constraint is considered alone, two corresponding $i_{d}$ values exist on the voltage constraint boundary for any given $i_{q}$ value in the flux-weakening region, corresponding to points $d$ and $e$ in Fig. 2. Obviously, reducing the magnitude of $i_{d}$, corresponding to point $d$ in Fig. 2, will minimize copper loss. The above observations provide the foundation for de- veloping flux-weakening current control with minimum copper loss in the following section. Moreover, if the motor is driven to remain on the intersection point between the current constraint boundary and the voltage constraint boundary, for example point $c$ in Fig. 2, then the output torque generated by the motor is continuously maximized, as is the operating range for the given inverter capacity.

\section{CURRENT LOOP DESIGN}

This section develops a current controller whose operating ranges can cover normal and flux-weakening operating regions. This outstanding feature comes from the ability of the proposed controller to allow re-selection of the d-axis current command to avoid overmodulation and to defer voltage saturation. Moreover, selecting d-axis current commands appropriately according to the suggestions outlined in Sections IV-C and IV-D, the current controller can drive the motor with minimum copper loss when $\mathrm{q}$-axis current command is inside the operable region, and with maximum output torque when q-axis current command exceeds the operable region.

\section{A. Current Controller Design}

The design objective of the proposed current controller is to defer output voltage saturation to as late as possible. The analyzes in Section III confirmed that the negative d-axis current can prevent early output voltage saturation and expand operating range to the flux-weakening region. Consequently, output voltage saturation can be prevented while preserving control stability, by including a term which contains the negative d-axis current command in the control laws represented by $v_{d}$ and $v_{q}$. By applying feedback linearization method [9], the current control laws embodied with the aforementioned idea are designed as

$$
\begin{aligned}
& v_{d}=L\left(\frac{R}{L} i_{d}^{*}-N \omega i_{q}-\lambda_{p d} e_{d}-\lambda_{i d} e_{d i}\right) \\
& v_{q}=L\left(N \omega i_{d}^{*}+\frac{R}{L} i_{q}^{*}-\lambda_{p q} e_{q}-\lambda_{i q} e_{q i}\right)+K N \omega
\end{aligned}
$$

where $i_{d}^{*}$ and $i_{q}^{*}$ are current command inputs; $e_{d}=i_{d}-i_{d}^{*}$ and $e_{q}=i_{q}-i_{q}^{*}$ are current control errors; $e_{d i}=\int e_{d} d t$ and $e_{q i}=$ $\int e_{q} d t$ are the integration of current control errors; $\lambda_{p d} \lambda_{i d}, \lambda_{p q}$, and $\lambda_{i q}$ are control gains. When the magnitude of the original computed output voltage vector exceeds that of the maximum output voltage vector, the term $L N \omega i_{d}^{*}$ in (9) makes it possible to directly re-select a negative $i_{d}^{*}$ to reduce the magnitude of the $v_{q}$ and to avoid output voltage saturation.

To analyze the control stability, we substitute control laws (8) and (9) into the electrical subsystem (1). As a result, the closed-loop error dynamics becomes

$$
\begin{aligned}
\frac{d}{d t}\left\{\begin{array}{c}
e_{d i} \\
e_{d} \\
e_{q i} \\
e_{q}
\end{array}\right\}= & {\left[\begin{array}{cccc}
0 & 1 & 0 & 0 \\
-\lambda_{d i} & -\left(\frac{R}{L}+\lambda_{d p}\right) & 0 & 0 \\
0 & 0 & 0 & 1 \\
0 & -N \omega & -\lambda_{q i} & -\left(\frac{R}{L}+\lambda_{q p}\right)
\end{array}\right] } \\
& \times\left\{\begin{array}{c}
e_{d i} \\
e_{d} \\
e_{q i} \\
e_{q}
\end{array}\right\}-L \frac{d}{d t}\left\{\begin{array}{c}
0 \\
i_{d}^{*} \\
0 \\
i_{q}^{*}
\end{array}\right\}
\end{aligned}
$$


According to the singular perturbation method [10], [11], owing to the two-time-scale property possessed by small electrical machines, the mechanical variable $\omega$ in (10) can be treated as a frozen value. Consequently, system (10) becomes a linear timeinvariant system, and numerous linear analysis techniques can be applied to analyze the system. Using the Laplace transformation method and choosing $\lambda_{d i}=\sigma_{d a} \sigma_{d b},\left(R / L+\lambda_{d p}\right)=$ $\sigma_{d a}+\sigma_{d b}, \lambda_{q i}=\sigma_{q a} \sigma_{q b}$, and $\left(R / L+\lambda_{q p}\right)=\sigma_{q a}+\sigma_{q b}$ allow us to express the relationships between command inputs and control errors as

$$
\begin{aligned}
E_{d}(s)= & -\frac{L s^{2}}{\left(s+\sigma_{d a}\right)\left(s+\sigma_{d b}\right)} I_{d}^{*}(s) \\
E_{q}(s)= & \frac{N \omega L s^{3}}{\left(s+\sigma_{d a}\right)\left(s+\sigma_{d b}\right)\left(s+\sigma_{q a}\right)\left(s+\sigma_{q b}\right)} I_{d}^{*}(s) \\
& -\frac{L s^{2}}{\left(s+\sigma_{q a}\right)\left(s+\sigma_{q b}\right)} I_{q}^{*}(s) .
\end{aligned}
$$

Clearly, stable poles of (11) and (12) can be assigned via properly chosen positive control gains, $\lambda_{p d}, \lambda_{i d}, \lambda_{p q}$, and $\lambda_{i q}$. Consequently, the control stability is guaranteed. Additionally, by applying the final value theorem, the steady-state error $\lim _{t \rightarrow \infty} e_{d}(t)=0$ even if $i_{d}^{*}$ is a ramp input, and $\lim _{t \rightarrow \infty} e_{q}(t)=0$ $t \rightarrow \infty$
even if $i_{d}^{*}$ is a parabolic input and $i_{q}^{*}$ is a ramp input.

\section{B. Digital Implementation}

Before introducing the methods to select $i_{d}^{*}$, the fundamental implementation methods of the proposed current controller should be introduced first.

Because the proposed controller is implemented digitally, the integral terms of control laws (8), (9) can be digitized as

$$
\begin{aligned}
& e_{d i}(k)=e_{d i}(k-1)+\left[i_{d}(k)-i_{d}^{*}(k)\right] T_{s} \\
& e_{q i}(k)=e_{q i}(k-1)+\left[i_{q}(k)-i_{q}^{*}(k)\right] T_{s}
\end{aligned}
$$

where $k$ denotes the sequence index, and $T_{s}$ represents the sampling period of the current loop. Rewrite control laws (8), (9) by isolating the $i_{d}^{*}$ terms, we get the digitized control laws as

$$
\begin{aligned}
v_{d}(k) & =D_{A}(k)+D_{B} i_{d}^{*}(k) \\
v_{q}(k) & =Q_{A a}(k)+Q_{A b} i_{q}^{*}(k)+Q_{B} i_{d}^{*}(k) \\
& =Q_{A}(k)+Q_{B} i_{d}^{*}(k)
\end{aligned}
$$

where

$$
\begin{aligned}
D_{A}(k)= & L\left\{-N \omega i_{q}(k)-\lambda_{p d} i_{d}(k)\right. \\
& \left.-\lambda_{i d}\left[e_{d i}(k-1)+i_{d}(k) T_{s}\right]\right\} ; \\
D_{B}= & L\left(\frac{R}{L}+\lambda_{p d}+\lambda_{i d} T_{s}\right) ; \\
Q_{A a}(k)= & L\left\{-\lambda_{p q} i_{q}(k)-\lambda_{i q}\left[e_{q i}(k-1)+i_{q}(k) T_{s}\right]\right\} \\
& +K N \omega ;
\end{aligned}
$$

$$
\begin{aligned}
Q_{A b} & =L\left(\frac{R}{L}+\lambda_{p q}+\lambda_{i q} T_{s}\right) ; \\
Q_{A}(k) & =Q_{A a}(k)+Q_{A b} i_{q}^{*}(k) ; \\
Q_{B} & =L N \omega .
\end{aligned}
$$

In the next two subsections, methods to select $i_{d}^{*}$ are introduced. Section IV-C describes the method to select $i_{d}^{*}$ to achieve minimum copper loss control when $i_{q}^{*}$ is inside the operable region. Section IV-D presents a method for selecting $i_{d}^{*}$ to maximize output torque control when $i_{q}^{*}$ exceeds the operable region.

\section{Inside the Operable Region}

Two rules can be applied for selecting $i_{d}^{*}$, depending on whether the output voltage vector calculated from the control laws exceeds the maximum output voltage vector.

Situation 1: $\left(\sqrt{v_{d}^{2}+v_{q}^{2}}>V_{\max }\right)$ : When the magnitude of the output voltage vector computed from (15) and (16) exceeds that of the maximum output voltage vector, $i_{d}^{*}$ is chosen to satisfy the maximum voltage equation $v_{d}(k)^{2}+v_{q}(k)^{2}=V_{\max }^{2}$. Substituting (15) and (16) into this maximum voltage equation, $i_{d}^{*}$ can be obtained by solving the following quadratic equation:

$$
\begin{gathered}
\left(D_{B}^{2}+Q_{B}^{2}\right) i_{d}^{*}(k)^{2}+2\left[D_{A}(k) D_{B}+Q_{A}(k) Q_{B}\right] i_{d}^{*}(k) \\
+\left[D_{A}(k)^{2}+Q_{A}(k)^{2}-V_{\max }^{2}\right]=0 .
\end{gathered}
$$

If the solutions of (17) are complex values with nonzero imaginary parts, the desired $i_{q}^{*}$ exceeds both the voltage constraint region and the operable region. Section IV-D suggests selections for $i_{d}^{*}$, while $i_{q}^{*}$ exceeds the operable region. Meanwhile, if (17) has two real solutions of $i_{d}^{*}$, the one with a smaller magnitude is chosen for minimum copper loss design as seen in (18), shown at the bottom of the page. Similarly, if the current vector combined by components $i_{q}^{*}$ and $i_{d}^{*}$ derived from (18) exceeds the current constraints and the operable region, the selection of $i_{d}^{*}$ needs to be dealt with according to the suggestions presented as follows in Section IV-D.

Situation 2: $\left(\sqrt{v_{d}^{2}+v_{q}^{2}} \leq V_{\max }\right)$ : When the magnitude of the output voltage vector is smaller than or equal to that of the maximum output voltage vector, $\left(\sqrt{v_{d}^{2}+v_{q}^{2}} \leq V_{\max }\right)$, output voltage saturation does not occur, and $i_{d}$ should return to a value that keeps the motor running while minimizing copper loss. $i_{d}^{*}$ is computed as

$$
\begin{gathered}
i_{d}^{*}(k+1)=i_{d}^{*}(k)+g_{1}\left(V_{\max }-\sqrt{v_{d}(k)^{2}+v_{q}(k)^{2}}\right) \\
\text { if }\left(i_{d}^{*}(k+1)>0\right) \quad i_{d}^{*}(k+1)=0
\end{gathered}
$$

where $g_{1}$ denotes a positive integral gain. The tuning rule of $i_{d}^{*}$ in (19) depicts that $i_{d}^{*}$ at the next cycle is modified by the integration of the voltage difference, $V_{\max }-\sqrt{v_{d}(k)^{2}+v_{d}(k)^{2}}$, and is limited to an upper bound at 0 . Basically, the tuning rule

$$
i_{d}^{*}(k)=\frac{-\left[D_{A}(k) D_{B}+Q_{A}(k) Q_{B}\right]+\sqrt{V_{\max }^{2}\left(D_{B}^{2}+Q_{B}^{2}\right)-\left[Q_{A}(k) D_{B}-D_{A}(k) Q_{B}\right]^{2}}}{\left(D_{B}^{2}+Q_{B}^{2}\right)}
$$




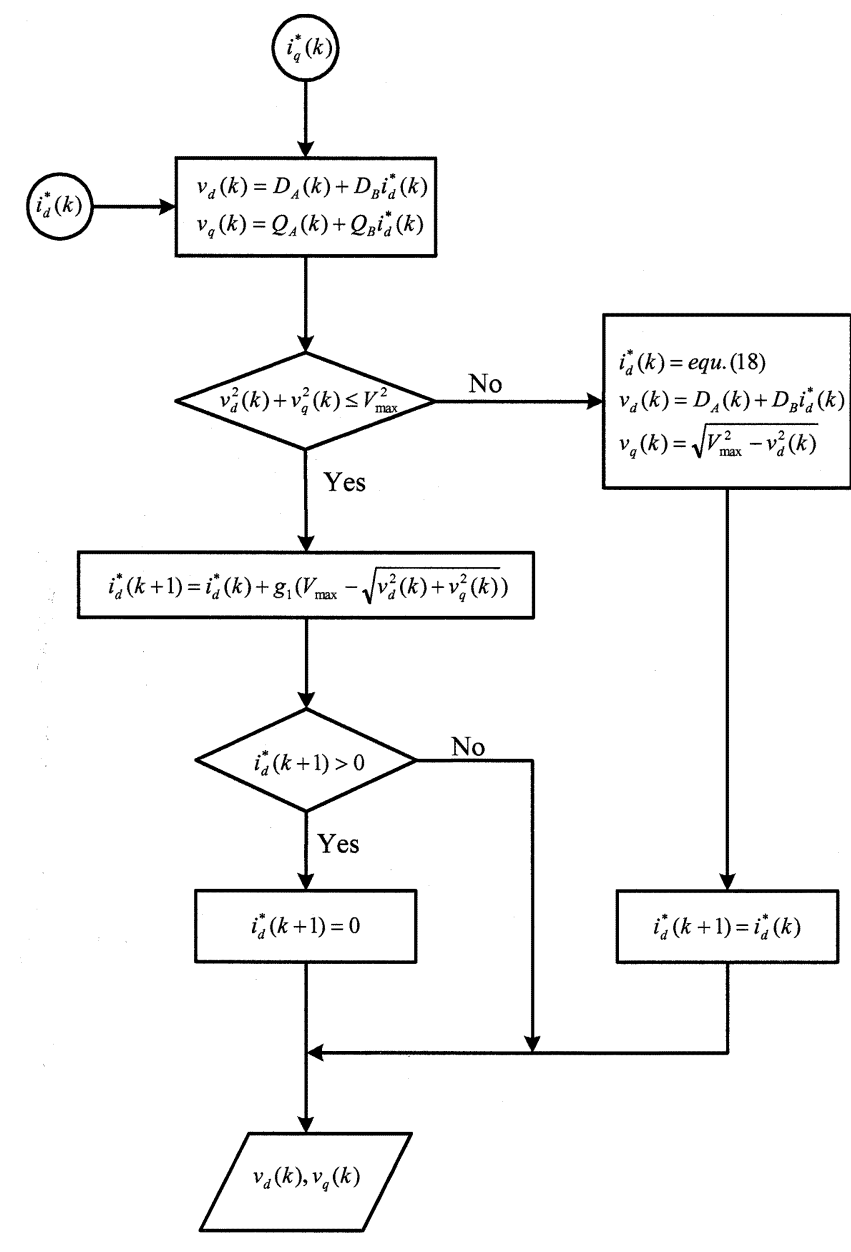

Fig. 3. Flow chart of the proposed current controller when $i_{q}^{*}$ remains within the operable region.

(19) will not affect the stability of the current control. However, during digital implementation the integral gain, $g_{1}$, must be upper bounded.

Fig. 3 illustrates a flow chart of the aforementioned current controller. The methods employed above for selecting $i_{d}^{*}$ focus on running a motor inside the operable region. Under such circumstances, the requested $i_{q}^{*}$ can always be achieved and the $i_{d}$ obtained by the controller will minimize copper loss. The following subsection discusses how to select $i_{d}^{*}$ when the requested $i_{q}^{*}$ exceeds the operable region.

\section{Outside the Operable Region}

The requested $i_{q}^{*}$ is unobtainable if no $i_{d}^{*}$ can move the operating point into the operable region. In other words, the requested $i_{q}^{*}$ is outside the operable region. Under such circumstances, a compromise approach is to select $i_{q}^{*}$ so as to maximize torque output in the operable region. Since the operating point that maximizes output torque can be limited by either

1) the current condition;

2) both current and voltage conditions together;

3) the voltage condition, the following three maximum torque control designs exist.

Situation 1: Limited by the Current Condition: If the operating point of the maximum torque control is only limited by the current condition, $i_{q}^{*}$ should clearly equal $I_{\max }$ and $i_{d}^{*}$ should be zero.

Situation 2: Simultaneously Limited by the Current Condition and the Voltage Condition: If the operating point of the maximum torque control is simultaneously limited by the current condition and the voltage condition, for example point $c$ in Fig. 2, $Q_{A}=Q_{A a}+Q_{A b} i_{q}^{*}$ can be substituted into (17), and (17) can then be solved combined with the constraint equation $i_{d}^{* 2}+i_{q}^{* 2}=I_{\max }^{2}$ to obtain the precise values of $i_{d}^{*}$ and $i_{q}^{*}$ that maximize output torque. However, due to the complex computation involved in solving the bi-quadratic equation, this approach is unsuitable for implementation in real-time control systems. An alternative method is suggested herein, in which the operating point gradually approaches the maximum torque point along the voltage constraint boundary. An $i_{d}^{*}$ tuning rule, similar to (19), is proposed to replace the precise $i_{d}^{*}$ value obtained from the bi-quadratic equation

$$
i_{d}^{*}(k)=i_{d}^{*}(k-1)+g_{2}\left(V_{\max }-\sqrt{v_{d}(k)^{2}+v_{q}(k)^{2}}\right)
$$

where $g_{2}$ denotes a positive integral gain; and $v_{d}(k)$ and $v_{q}(k)$ represent the output voltages for the initial $i_{d}^{*}$ and $i_{q}^{*}$ at the beginning of that cycle. Moreover, $i_{q}^{*}$ must be re-calculated each cycle to satisfy the output voltage equation, $v_{d}^{2}+v_{q}^{2}=V_{\max }^{2}$. Although this method cannot always maximize torque output, it is capable of maximizing torque output when the system reaches its steady state.

Situation 3: Limited by the Voltage Condition: When the operating point of the maximum torque control is only limited by the voltage condition, the precise values of $i_{d}^{*}$ and $i_{q}^{*}$ for maximizing output torque can be directly obtained from (7) as

$$
\begin{aligned}
& i_{d}^{*}=\frac{-K L N^{2} \omega^{2}}{R^{2}+L^{2} N^{2} \omega^{2}} \\
& i_{q}^{*}=-\frac{K N \omega R}{R^{2}+L^{2} N^{2} \omega^{2}}+\operatorname{sgn}(\omega) \frac{V_{\max }}{\sqrt{R^{2}+L^{2} N^{2} \omega^{2}}} .
\end{aligned}
$$

However, to simplify the computation, similar rules for selecting $i_{d}^{*}$ and $i_{q}^{*}$ as in Situation 2 are adopted, except that a minimum value of $i_{d}^{*}$ is added to tuning rule (20). From (7), the minimum value of $i_{d}^{*}$ for maximizing output torque is

$$
i_{d \min }^{*}=\frac{-K L N^{2} \omega^{2}}{R^{2}+L^{2} N^{2} \omega^{2}} .
$$

Similarly, once $i_{d}^{*}$ is selected for a given cycle, $i_{q}^{*}$ must also be re-computed for that cycle to satisfy the output voltage equation, $v_{d}^{2}+v_{q}^{2}=V_{\max }^{2}$.

To summarize the discussions in Sections IV-C and IV-D, a complete flow chart for realizing the proposed current controller is displayed in Fig. 4. Because $i_{d}^{*}$ and $i_{q}^{*}$ are bounded inputs, the proposed current laws (8), (9) can achieve stable current control. Additionally, following the proposed current command selection rules, the motor operation always minimizes copper loss.

\section{EXPERIMENTAL RESULTS}

Experiments are performed to verify the proposed current controller. Because flux-weakening control can expand the operating range of the motor, high-speed experiments under a des- 


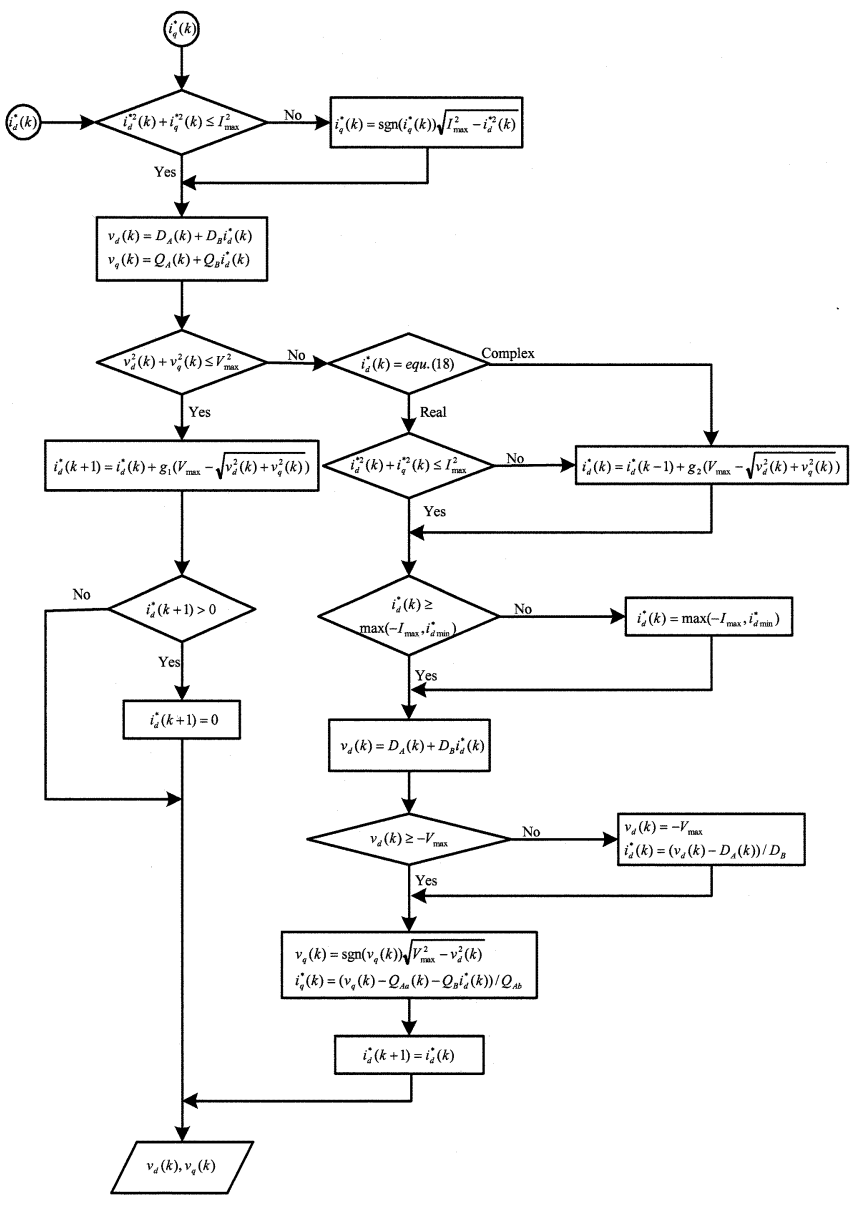

Fig. 4. Complete flow chart of the proposed current controller.

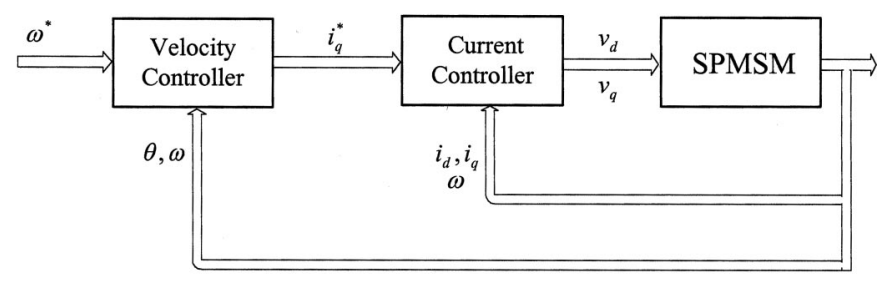

Fig. 5. Block diagram of the motor drive.

ignated inverter capacity are helpful for examining the performance of the automatic flux-weakening control of the proposed current controller. A simple PI velocity controller with $1 \mathrm{kHz}$ sampling rate is implemented in these experiments. Meanwhile, the proposed current controller is implemented based on the flow chart in Fig. 4 with a sampling rate of $5 \mathrm{kHz}$. The block diagram of the motor drive is illustrated in Fig. 5, where $\theta$ denotes the rotor position.

Fig. 6 illustrates the experimental setup, which includes a Sinano \#7CB30-2SE6F permanent magnet synchronous motor with ratings and identified parameters as listed in Table I, the inverter of a Micro Trend UT90 Drive, a proprietary control card made in-house, and a PC. The control card converts the analog phase current measurements into digital signals, decodes the encoder signals, and generates SVPWM switching signals to control the inverter. The PC is used to compute the control al-

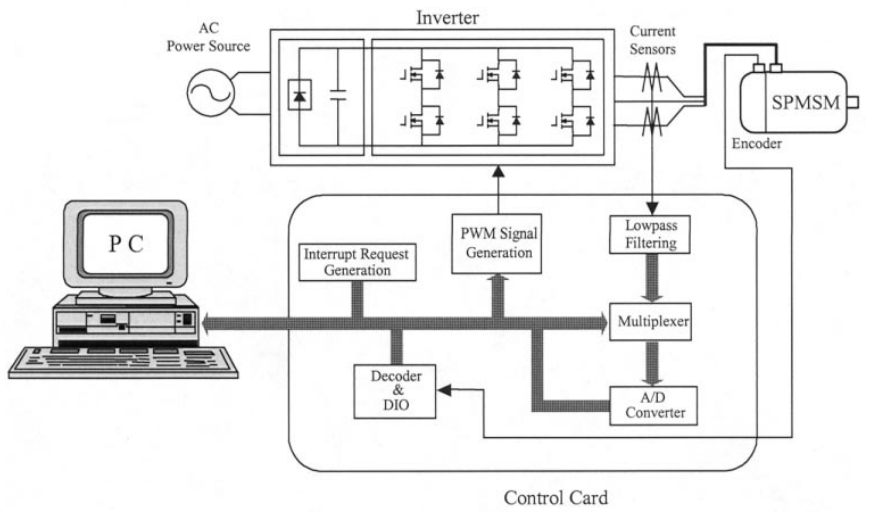

Fig. 6. Experimental setup.

TABLE I

RATINGS AND IDENTIFIED PARAMETERS OF THE SINANO \#7CB30-2SE6F MOTOR

\begin{tabular}{ll}
\hline Ratings & $\mathrm{P}_{\text {rated }}=300[\mathrm{~W}], \mathrm{V}_{\text {rated }}=200[\mathrm{~V}], \omega_{\text {rated }}=3000[\mathrm{rpm}]$, \\
& $\mathrm{I}_{\text {phase_rated }}=2[\mathrm{~A}], \mathrm{I}_{\text {phase_peak }}=6[\mathrm{~A}]$. \\
\hline Pole pair & $N=4$ \\
Resistance & $R=3.55[\Omega]$ \\
Inductance & $L=5.92 \times 10^{-3}[\mathrm{H}]$ \\
Magnet constant & $K=5.795 \times 10^{-2}[\mathrm{~V} \cdot \mathrm{sec} / \mathrm{rad}]$ \\
Rotor inertia & $J=6.45 \times 10^{-5}\left[\mathrm{~kg} \cdot \mathrm{m}^{2}\right]$ \\
Viscous damping coefficient & $B=8 \times 10^{-5}[\mathrm{~N} \cdot \mathrm{m} \cdot \mathrm{sec} / \mathrm{rad}]$ \\
Column friction coefficient & $C=1.738 \times 10^{-2}[\mathrm{~N} \cdot \mathrm{m}]$ \\
\hline
\end{tabular}

gorithms and the coordinate transformations among the vector space, the stator reference frame, and the rotor reference frame.

Three experiments are performed, in which the DC link voltage of the inverter, $V_{D C}$, is set at $140 \mathrm{~V}$, such that the motor drive will enter into the flux-weakening region when the motor is operated at high speeds. According to the motor parameters listed in Table I and the given $V_{D C}$, the minimum speed that needs flux weakening can be calculated at $3311 \mathrm{rpm}$. Thus, flux-weakening control becomes necessary when the operating speed exceeds $3311 \mathrm{rpm}$. Additionally, the maximum current limit of inverter, $I_{\max }$, is set at $2 \mathrm{~A}$.

First, a smooth velocity tracking experiment is performed, and the experimental results are shown in Fig. 7. In this experiment, velocity command, as illustrated in Fig. 7(a), is a smooth trapezoidal trajectory that ranges between $3000 \mathrm{rpm}$ and 4000 rpm. Under this velocity command, according to the experimental results, $i_{q}^{*}$ is always inside the operable region. Consequently, the computation of the current controller follows the flow chart illustrated in Fig. 3. As exhibited in Fig. 7(c), the response of $i_{d}$ is negative in the high-speed range and returns to zero in the low speed range. Moreover, the output voltage ratio, $\rho_{V}=\sqrt{v_{d}^{2}+v_{q}^{2}} / V_{\max }$, closely approaches one in the flux-weakening region, as shown in Fig. 7(b). The analysis in Section III indicates that the response of $i_{d}$ displayed in Fig. 7(c) always minimizes copper loss.

Further experiments are conducted by changing the initial velocity command into a step input, and the experimental results 

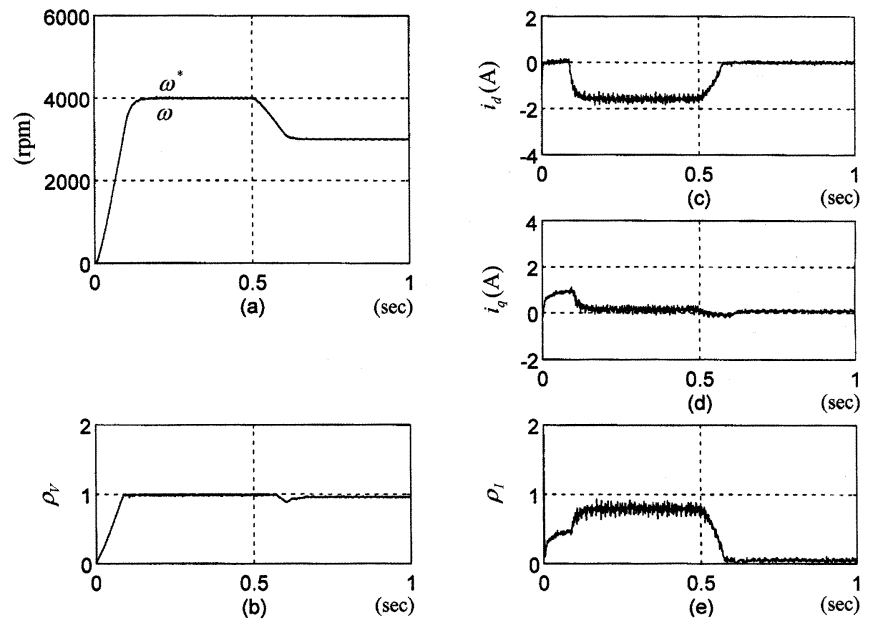

Fig. 7. Results of the flux-weakening operation experiment for the smooth velocity tracking command. (a) velocity command and velocity; (b) output voltage ratio $\rho_{V}=\sqrt{v_{d}^{2}+v_{q}^{2}} / V_{\max }$; (c) d-axis current; (d) q-axis current; (e) current ratio $\rho_{I}=\sqrt{i_{d}^{2}+i_{q}^{2}} / I_{\max }$.
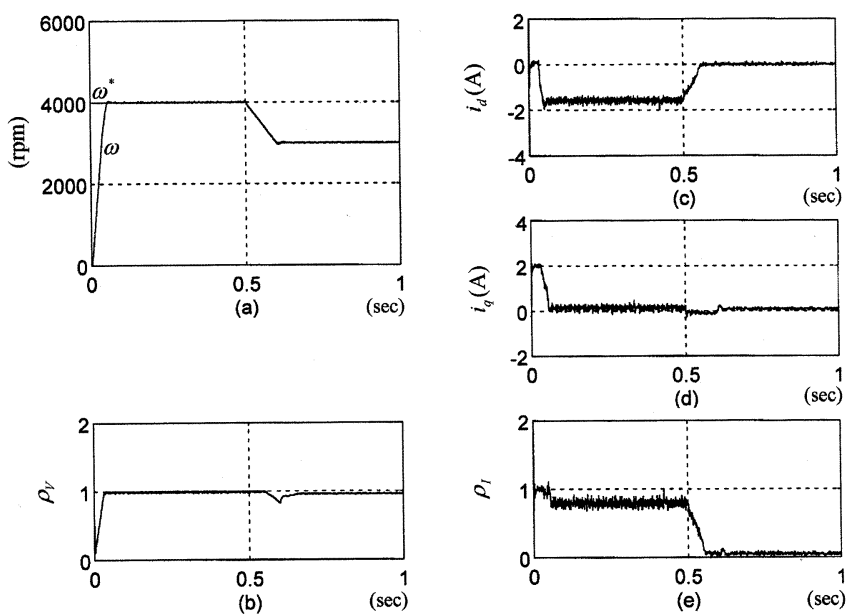

Fig. 8. Results of the flux-weakening operation experiment for the velocity tracking command whose initial command is a step. (a) velocity command and velocity; (b) output voltage ratio $\rho_{V}=\sqrt{v_{d}^{2}+v_{q}^{2}} / V_{\max }$; (c) d-axis current; (d) q-axis current; (e) current ratio $\rho_{I}=\sqrt{i_{d}^{2}+i_{q}^{2}} / I_{\max }$.

are presented in Fig. 8. Maximum output torque control limited only by the current condition, as discussed in Section IV-D, Situation 1 , present in the initial accelerated region. As shown in Fig. 8(d), (e), $i_{q}$ is close to $2 \mathrm{~A}$ in the initial accelerated region, and the current ratio, $\rho_{I}=\sqrt{i_{d}^{2}+i_{q}^{2}} / I_{\max }$, also closely approaches one in that region.

An additional experiment is conducted to determine whether the proposed current controller can efficiently employ the maximum inverter capacity, $V_{\max } I_{\max }$, to drive the motor. Fig. 9 shows the experimental results. The initial step velocity command is up to $5000 \mathrm{rpm}$, as exhibited in Fig. 9(a), which is outside the operable region of the motor for the given inverter capacity. Under such circumstances, according to previous an-
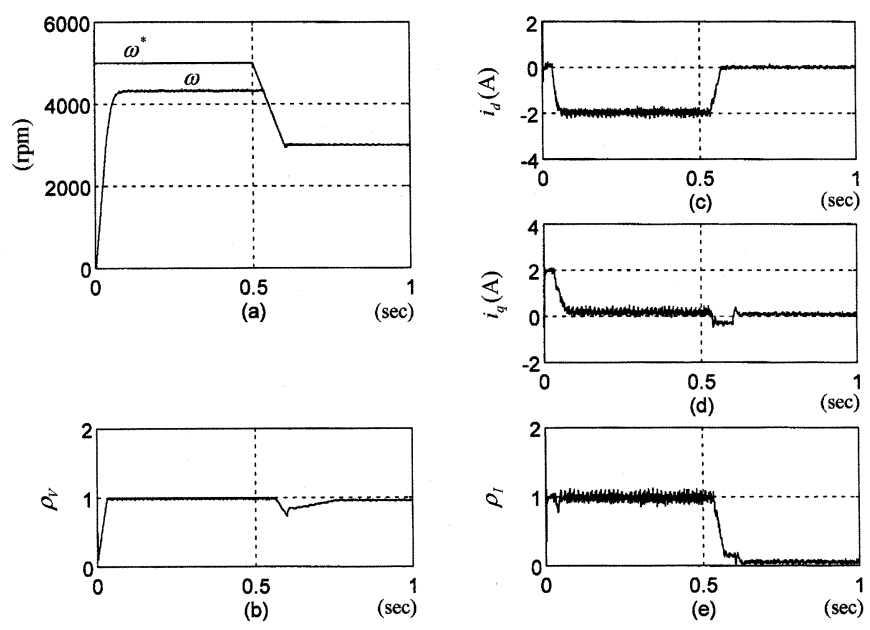

Fig. 9. Results of the flux-weakening operation experiment for the velocity tracking command whose high speed part exceeds the operable range. (a) velocity command and velocity; (b) output voltage ratio $\rho_{V}=\left(\sqrt{v_{d}^{2}+v_{q}^{2}} / V_{\max }\right)$; (c) d-axis current; (d) q-axis current; (e) current ratio $\rho_{I}=\left(\sqrt{i_{d}^{2}+i_{q}^{2}} / I_{\max }\right)$.

alyzes, the proposed current controller should perform a maximum output torque control. Fig. 9(b), (e) clearly displays that the maximum output torque control in the high-speed region is simultaneously limited by both the current and the voltage conditions, as discussed in Section IV-D, Situation 2, where the output voltage ratio, $\rho_{V}$, and the current ratio, $\rho_{I}$, are both close to one. Because the flux-weakening current always minimizes copper loss in the motor, the experimental results imply that the current controller can efficiently employ the maximum inverter capacity to drive the motor even in the flux-weakening region. Consequently, the highest speed in Fig. 9(a) is the highest operating speed for the motor driver for the given inverter capacity.

\section{CONCLUSION}

This study presents a novel current loop design method that can achieve automatic flux-weakening control with minimum copper loss for SPMSM. The outstanding characteristics of the proposed current controller come from the control law (9), which contains a $L N \omega i_{d}^{*}$ term. This term allows $i_{d}^{*}$ to be directly reselected to avoid output voltage saturation while preserving control stability. As a result, the proposed current controller can achieve an active and stable flux-weakening control. Moreover, the proposed current controller always keeps the output voltage vector on the voltage constraint boundary in the flux-weakening region, indicating that the flux-weakening current is constantly optimized for minimizing copper loss. Additionally, when the requested $i_{q}^{*}$ exceeds the operable region, the proposed current controller can drive the motor to achieve maximum output torque control. Experimental results demonstrated the feasibility of the proposed current controller, and verified that the proposed controller can efficiently employ the inverter capacity to drive the motor to the maximum operating speed possible for a given inverter capacity. 


\section{REFERENCES}

[1] S. Morimoto, Y. Takeda, T. Hirasa, and K. Taniguchi, "Expansion of operating limits for permanent magnet by current vector control considering inverter capacity," IEEE Trans. Ind. Applicat., vol. 26, pp. 866-871, Sept./Oct. 1990.

[2] S. Morimoto, Y. Takeda, and T. Hirasa, "Flux-weakening control method for surface permanent magnet synchronous motors," in Proc. IPEC'90 Conf., Tokyo, Japan, 1990, pp. 942-949.

[3] J. Shi and Y.-S. Lu, "Field-weakening operation of cylindrical permanent-magnet motors," in Proc. IEEE Int. Conf. Contr. Applicat., Dearborn, MI, Sept. 1996, pp. 848-864.

[4] Y.-S. Verl and M. Bodson, "Torque maximization for permanent magnet synchronous motors," IEEE Trans. Contr. Syst. Tech., vol. 6, pp. 740-745, Nov. 1998

[5] S. D. Sudhoff, K. A. Corzine, and H. J. Hegner, "A flux-weakening strategy for current-regulated surface-mounted permanent-magnet machine drives," IEEE Trans. Energy Conv., vol. 10, pp. 431-437, Sept. 1995.

[6] J.-H. Song, J.-M. Kim, and S.-K. Sul, "A new robust SPMSM control to parameter variations in flux weakening region," Proc. IEEE, vol. 2, pp. 1193-1198, Aug. 1996.

[7] K.-M. Kim and S.-K. Sul, "Speed control of interior permanent magnet synchronous motor drive for flux weakening operation," Ind. Applicat. Conf., vol. 1, pp. 216-221, 1995.

[8] J.-J. Chen and K.-P. Chin, "Automatic flux-weakening control of permanent magnet synchronous motors using a reduced-order controller," IEEE Trans. Power Electron., vol. 15, pp. 881-890, Sept. 2000.

[9] J.-J. E. Slotine and W. Li, Applied Nonlinear Control. Englewood Cliffs, NJ: Prentice-Hall, 1991.

[10] P. V. Kokotovic, H. K. Khalil, and J. O'Reilly, Singular Perturbation Methods in Control: Analysis and Design. London, U.K.: Academic, 1986 , ch. 1.
[11] H. K. Khalil, Nonlinear Systems, 2nd ed. Englewood Cliffs, NJ: Prentice-Hall, 1996, ch. 9.

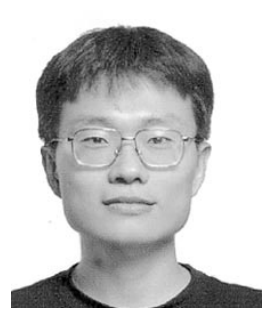

Jiunn-Jiang Chen was born in Kaohsiung, Taiwan, R.O.C., in 1972. He received the B.S. and the Ph.D. degrees from National Chiao-Tung University, Hsinchu, Taiwan, in 1995 and 2000, respectively.

$\mathrm{He}$ then joined the Aetas System Incorporated Company to work in the field of motor driving technique and imaging technique on imaging apparatus. His current research interests include power electronics, identification, signal processing, and electrical machine control.

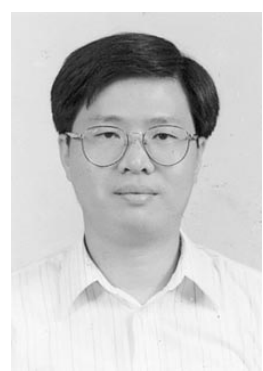

Kan-Ping Chin (S'89-M'92) was born in Taipei, Taiwan, R.O.C., on June 30, 1960. He received the B.S. degree in mechanical engineering from National Taiwan University, Taiwan, R.O.C., in 1982 and the M.S. and Ph.D. degrees from the Massachusetts Institute of Technology, Cambridge, in 1988 and 1991, respectively.

$\mathrm{He}$ was a faculty member at the National Chiao-Tung University, Taiwan, from 1992 to 2002. His premature and unanticipated death on February 8, 2002 was a loss not only to those who loved and admired him but to his burgeoning research on control of servo motors and microelectromechanical systems (micromotors). 